九州大学学術情報リポジトリ

Kyushu University Institutional Repository

\title{
ON ASSOCIATIVE SHORTEST PATH PROBLEMS
}

Maruyama, Yukihiro

Department of Mathematics, Faculty of Liberal Arts, Nagasaki University

https://doi.org/10.5109/13462

出版情報: Bulletin of informatics and cybernetics. 29 (1), pp.67-81, 1997-03. Research Association of Statistical Sciences

バージョン :

権利関係 : 


\title{
ON ASSOCIATIVE SHORTEST PATH PROBLEMS
}

\author{
By
}

Yukihiro MaRUYama *

\begin{abstract}
We consider a wide class of shortest path problems in acyclic digraphs. In the problems, the length of a path is defined by using an associative binary operation. We derive recursive equations in dynamic programming for the problems, which involve additive, multiplicative, multiplicative-additive, minimum and fractional shortest path problems. A necessary and sufficient condition and two sufficient conditions for the recursive equation to have a solution are given because for all problems the recurcive equation does not hold. In case the equation has a solution, a sequence which converges to the solution is proposed.
\end{abstract}

Keywords and Phrases: acyclic digraphs; associative binary operation; directed network; shortest path problems.

\section{Introduction}

In this paper, we are concerned with the problem of finding a path of minimum or maximum length from a single origin node to a few destination nodes in a directed network $G(V, A)$, where $V$ is a finite set of nodes and $A$ is a finite set of directed arcs. Let node 1 be the origin node and let $S^{t}$ be the set of the destination nodes: $S^{t}=\left\{i^{*} \in V \mid D\left(i^{*}\right)=\emptyset\right\}$, where $D(i)=\{j \in V \mid(i, j) \in A\}$. With each arc $(i, j) \in A$ and each destination node $i^{*} \in S^{t}$ we associate arc length (or cost) $t_{i j} \in R$ and terminal reward $k\left(i^{*}\right) \in R$, respectively. A path $p$ from $i$ to $i^{*} \in S^{t}$ is denoted by

$$
p=\left(i, j_{1}, j_{2}, \ldots, j_{k-1}, j_{k}, i^{*}\right),
$$

where

$$
i, j_{1}, j_{2}, \cdots, j_{k-1}, j_{k} \in V \backslash S^{t}, i^{*} \in S^{t}, \quad\left(i, j_{1}\right),\left(j_{1}, j_{2}\right), \cdots,\left(j_{k-1}, j_{k}\right),\left(j_{k}, i^{*}\right) \in A .
$$

A length of the path $p$ is defined by

$$
t_{i j_{1}} \circ t_{j_{1} j_{2}} \circ \cdots \circ t_{j_{k-1} j_{k}} \circ t_{j_{k} i^{*}} \circ k\left(i^{*}\right)
$$

* Department of Mathematics, Faculty of Liberal Arts, Nagasaki University, 1-14, Bunkyomachi, Nagasaki 852, Japan 
where $\circ: R \times R \rightarrow R$ is an associative binary operation: $(x \circ y) \circ z=x \circ(y \circ z)$.

The objective is to find a path of minimum or maximum length which starts at the node 1 and ends at some $i^{*} \in S^{t}$ :

$$
\underset{p}{\mathrm{Opt}}\left[t_{1 j_{1}} \circ t_{j_{1} j_{2}} \circ \cdots \circ t_{j_{k-1} j_{k}} \circ t_{j_{k} i^{*}} \circ k\left(i^{*}\right)\right]
$$

where Opt denotes either Max or min, optimizer and $p$ is a path from 1 to $i^{*} \in S^{t}$ :

$$
p=\left(1, j_{1}, j_{2}, \ldots, j_{k}, i^{*}\right)
$$

Here we note that optimization in (1) is taken for all paths from 1 to some $i^{*} \in S^{t}$. We call this problem associative shortest path problem and denote it by (ASP).

Throughout this paper, we suppose that network $G(N, A)$ contains no cycles and that every node $i \in V$ is connected to all destination nodes with a path.

If $S^{t}$ is a single node set: $S^{t}=\{N\}$, then the terminal reward $k\left(i^{*}\right)$ is meaningless. So, in this case, we consider the following problem:

$$
\underset{p}{\mathrm{Opt}}\left[t_{1 j_{1}} \circ t_{j_{1} j_{2}} \circ \cdots \circ t_{j_{k-1} j_{k}} \circ t_{j_{k} N}\right]
$$

where

$$
p=\left(1, j_{1}, j_{2}, \ldots, j_{k}, N\right) .
$$

Let $R(\circ)$ denote a real number satisfying that

$$
t \circ R(\circ)=t \quad \text { for all } t \in T,
$$

where $T=\left\{t_{i j} \circ \ldots \circ t_{n i^{*}} \mid\left(i, j, \ldots, n, i^{*}\right):\right.$ path, $\left.i^{*} \in S^{t}, i \notin S^{t}\right\}$.

Then this problem are also obtained by putting $k\left(i^{*}\right)=R(0)$ in the problem (ASP).

In case the binary operation $\circ$ in (1) is additive, the problem (ASP) reduces to the well-known problem, which we call the additive (shortest path) problem. In many monographs and papers, the additive problem has been effectively solved by dynamic programming (see, for example Bellman, Esogubue and Nabeshima (1982), Dreyfus (1969)).

On the other hand, in case the operation is multiplicative, Iwamoto (1987), Smith (1991) and Sniedovich (1992) derived the recursive equation to solve the shortest path problem. Moreover, Iwamoto (1987) and Sniedovich (1992) also studied the case $\circ=$ $\wedge, \vee$, where for any real numbers $a$ and $b$

$$
a \wedge b=\min (a, b), \quad a \vee b=\operatorname{Max}(a, b) .
$$

Recently, Maruyama (1996) solved a wide class of shortest path problems which contains the additive and multiplicative problems, but does not contain the case $\circ=\wedge, \vee$.

The problem (ASP) in this paper involves not only the additive, multiplicative, minimum and maximum problems but also many other problems, for example, multiplicativeadditive and fractional ones. We will derive the recursive equations for the problem 
(ASP). It is noted that the recursive equation does not necessarily hold without conditions.

So, in Section 2, we give a necessary and sufficient condition and two sufficient conditions for the recurcive equation in (ASP) to have a solution. Furthermore, we show the uniqueness of a solution of the equation if it exists. In Section 3 , we give a sequence which converges to the unique solution in case it exists.

\section{Existence and Uniquness}

To solve the problem (ASP), we imbed it into the following family of problems:

$$
\begin{aligned}
f_{i} & =\underset{p}{\operatorname{Opt}}\left[t_{i j_{1}} \circ t_{j_{1} j_{2}} \circ \cdots \circ t_{j_{k-1} j_{k}} \circ t_{j_{k} i^{*}} \circ k\left(i^{*}\right)\right] \quad i \notin S^{t}, \\
f_{i^{*}} & =k\left(i^{*}\right) \quad i^{*} \in S^{t}
\end{aligned}
$$

where optimization in (3) is taken for all path $p$ from $i$ to $i^{*} \in S^{t}$;

$$
p=\left(i, j_{1}, j_{2}, \ldots, j_{k-1}, j_{k}, i^{*}\right)
$$

In case $\circ=+$ and $S^{t}=\{N\}$, it is well known that $\left\{f_{i} \mid i \in V\right\}$ is a solution of the recursive equation

$$
g_{i}=\underset{j \in D(i)}{\operatorname{Opt}}\left[t_{i j}+g_{j .}\right] \quad i \neq N, \quad g_{N}=0
$$

On the other hand, for all associative operation, $\left\{f_{i} \mid i \in V\right\}$ does not satisfy the following recursive equation (see Example 2.8):

$$
g_{i}=\underset{j \in D(i)}{\operatorname{Opt}}\left[t_{i j} \circ g_{j}\right] \quad i \notin S^{t}, \quad g_{i^{*}}=k\left(i^{*}\right) \quad i^{*} \in S^{t} .
$$

THEOREM 2.1 (NECESSARY AND SUFFICIENT CONDITION). The set of the optimal values $\left\{f_{i}, i \in V\right\}$ defined by (3) is a solution of (4) if and only if the problem (ASP) satisfies the following condition:

$$
<R_{1}>\underset{j \in D(i)}{\operatorname{Opt}}\left[t_{i j} \circ f_{j}\right] \leq \operatorname{Opt}\left\{t_{i j} \circ a \mid a \in A_{j}\right\} \quad \forall j \in D(i), \forall i \notin S^{t}
$$

where $\leq$ denotes $\leq$ and $\geq$ in case Opt $=\min$ and Opt $=$ Max, respectively, $A_{j}=$ $\left\{t_{j k} \circ \ldots \circ t_{n i^{*}} \circ k\left(i^{*}\right) \mid\left(j, k, \ldots, n, i^{*}\right): p a t h, i^{*} \in S^{t}\right\}$ for $j \notin S^{t}$ and $A_{i^{*}}=\left\{k\left(i^{*}\right)\right\}$ for $i^{*} \in S^{t}$.

Proof. It suffices to show only the case Opt $=$ min. Similarly, the case Opt $=\mathrm{Max}$ is proved. We assume that the problem (ASP), satisfies the condition $<R_{1}>$. Let $i \notin S^{t}$ be given but arbitrary. Let $\left(i, j, k, \cdots, n, i^{*}\right)$ be any path from $i$ to $i^{*} \in S^{t}$. Then

$$
t_{i j} \circ\left(t_{j k} \circ \cdots \circ t_{n i *} \circ k\left(i^{*}\right)\right) \geq \min \left\{t_{i j} \circ a \mid a \in A_{j}\right\} .
$$


Further, from the condition $\left\langle R_{1}\right\rangle$, we have

$$
\min \left\{t_{i j} \circ a \mid a \in A_{j}\right\} \geq \min _{j \in D(i)}\left[t_{i j} \circ f_{j}\right]
$$

for all $j \in D(i)$. It follows from (5) and (6) that

$$
f_{i} \geq \min _{j \in D(i)}\left[t_{i j} \circ f_{j}\right]
$$

On the other hand, it is easily proved that

$$
f_{i} \leq \min _{j \in D(i)}\left[t_{i j} \circ f_{j}\right]
$$

Hence from (7), we get

$$
f_{i}=\min _{j \in D(i)}\left[t_{i j} \circ f_{j}\right]
$$

Conversely, suppose that $\left\langle R_{1}>\right.$ does not hold: there exist $i^{\prime} \notin S^{t}$ and $j^{\prime} \in D\left(i^{\prime}\right)$ such that

$$
\min _{j \in D\left(i^{\prime}\right)}\left[t_{i^{\prime} j} \circ f_{j}\right]>\min \left\{t_{i^{\prime} j^{\prime}} \circ a \mid a \in A_{j^{\prime}}\right\} .
$$

From this and the inequality

$$
\min \left\{t_{i^{\prime} j^{\prime}} \circ a \mid a \in A_{j^{\prime}}\right\} \geq f_{i^{\prime}}
$$

we get

$$
\min _{j \in D\left(i^{\prime}\right)}\left[t_{i^{\prime} j} \circ f_{j}\right]>f_{i^{\prime}} .
$$

Consequently, $f_{i^{\prime}}$ does not satisfy (4).

Corollary 2.2 (Sufficient CONDition). Let the problem (ASP) satisfy the following condition:

$$
<R_{2}>t_{i j} \circ f_{j} \leq \operatorname{Opt}\left\{t_{i j} \circ a \mid a \in A_{j}\right\} \quad \forall j \in D(i), \quad \forall i \notin S^{t} .
$$

Then $\left\{f_{i} \mid i \in V\right\}$ is a solution of (4).

Proof. The condition $<R_{2}>$ implies $<R_{1}>$.

Corollary 2.3 (Sufficient CONDition). Suppose that the problem (ASP) satisfies the following condition:

$$
<R_{3}>\quad b, c \in A_{j}, \quad b \leq c \Longrightarrow t_{i j} \circ b \leq t_{i j} \circ c \quad \forall j \in D(i), \quad \forall i \notin S^{t} .
$$

Then $\left\{f_{i} \mid i \in V\right\}$ is a solution of (4). 
Proof. Let $i \notin S^{t}, j \in D(i)$ be given but arbitrary. Then it holds that

$$
f_{j} \leq a \text { for all } a \in A_{j} .
$$

Hence it follows from the condition $\left\langle R_{3}>\right.$ that

$$
t_{i j} \circ f_{j} \leq t_{i j} \circ a \text { for all } a \in A_{j} .
$$

From this, we get

$$
t_{i j} \circ f_{j} \leq \operatorname{Opt}\left\{t_{i j} \circ a \mid a \in A_{j}\right\} \quad \text { for all } j \in D(i), i \notin S^{t},
$$

thus, $<R_{3}>$ implies $<R_{2}>$.

REMARK. In case $S^{t}=\{N\}$, we imbed the problem (ASP) into the following family of problems in place of $(3)$ :

$$
\begin{aligned}
f_{i} & =\underset{p}{\operatorname{Opt}}\left[t_{i j_{1}} \circ t_{j_{1} j_{2}} \circ \cdots \circ t_{j_{k-1} j_{k}} \circ t_{j_{k} N}\right] \quad i \neq N, \\
f_{N} & =R(\circ) .
\end{aligned}
$$

So, in this case we obtain the following recursive equation instead of (4):

$$
f_{i}=\underset{j \in D(i)}{\operatorname{Opt}}\left[t_{i j} \circ f_{j}\right] \quad i \neq N, \quad f_{N}=R(\circ)
$$

REMARK. If the associative operation $\circ$ is monotone on $R$ at each $a \in R: b, c \in$ $R, b<c \Longrightarrow a \circ b \leq a \circ c$, then the problem (ASP) satisfies the condition $<R_{3}>$. Hence in this case, from Corollary 2.3, we see that $\left\{f_{i} \mid i \in V\right\}$ is a solution of (4).

For example, addition is monotone on $R$ at each $a \in R$. Therefore the recursive equation (4) holds for the additive problem.

REMARK. The implication is

$$
<R_{3}>\Longrightarrow<R_{2}>\Longrightarrow<R_{1}>\text {. }
$$

It should be noted that a gap exists between these conditions.

Example 2.4 (Minimum problem). We consider the case $\circ=\wedge$. Since this operation is monotone on $R$ at each $a \in \hbar$, we have

$$
f_{i}=\underset{j \in D(i)}{\operatorname{Opt}}\left[t_{i j} \wedge f_{j}\right] \quad i \notin S^{t}, \quad f_{i^{*}}=k\left(i^{*}\right) \in R \quad i^{*} \in S^{t} .
$$

Put

$$
R(\wedge)=\operatorname{Max}\left\{t_{i j} \mid i, j \in V,(i, j) \in A\right\} .
$$

Then $R(\wedge)$ satisfies (2). Hence in case $S^{t}=\{N\}$, from (8) we obtain

$$
f_{i}=\underset{j \in D(i)}{\operatorname{Opt}}\left[t_{i j} \wedge f_{j}\right] \quad i \neq N, \quad f_{N}=R(\wedge) .
$$


On the other hand, replacing $\wedge$ by $\vee$ yields Maximum problem. Similarly, the results corresponding to (9) and (10) holds for this problem, where $R(\wedge)$ in (10) are replaced with

$$
R(\vee)=\min \left\{t_{i j} \mid i, j \in V,(i, j) \in A\right\}
$$

Example 2.5 (Multiplicative-additive problem). In this example, we consider the following associative operation: $a \circ b=a b+a+b$. We suppose that

$$
t_{i j}+1 \geq 0 \quad \forall i, j \notin S^{t}
$$

Then the problem (ASP) satisfies the condition $<R_{3}>$. Hence from Corollary 2.3, we have

$$
f_{i}=\underset{j \in D(i)}{\operatorname{Opt}}\left[\left(t_{i j}+1\right)\left(f_{j}+1\right)-1\right] \quad i \notin S^{t}, \quad f_{i^{*}}=k\left(i^{*}\right) \in R \quad i^{*} \in S^{t} .
$$

For $0(=R(0)),(2)$ holds. Hence in case $S^{t}=\{N\}$, from (8), we obtain

$$
f_{i}=\underset{j \in D(i)}{\operatorname{Opt}}\left[\left(t_{i j}+1\right)\left(f_{j}+1\right)-1\right], \quad i \neq N, \quad f_{N}=0 .
$$

REMARK. Iwamoto (1996) has introduced the operation : $a \circ b=a b+a+b$ in associative dynamic programs. Now let us propose the following one parameter associative operation:

$$
a \circ b=f(s ; a, b)=a b+s(a+b+s-1) .
$$

Then the operation : $a \circ b=a b+a+b$ and the multiplication are unified into it. If we suppose

$$
t_{i j}+s \geq 0 \quad \forall i, j \notin S^{t},
$$

we obtain recursive equations with the parameter $s$ which are similar to (12), (13).

Example 2.6 (Fractional Problem). Let us consider the following asssociative operation: $a \circ b=(a+b) /(1+a b)$. We assume that

$$
\left\{\begin{aligned}
k\left(i^{*}\right) \geq 0 & \text { for } i^{*} \in S^{t}, \\
t_{i j} \geq 0 & \text { for } i \notin S^{t}, j \in D(i), \\
1 \geq t_{i j} \geq 0 & \text { for } j \notin S^{t},
\end{aligned}\right\} \text { or }\left\{\begin{aligned}
k\left(i^{*}\right) \leq 0 & \text { for } i^{*} \in S^{t} \\
t_{i j} \leq 0 & \text { for } i \notin S^{t}, j \in D(i), \\
-1 \leq t_{i j} \leq 0 & \text { for } j \notin S^{t}
\end{aligned}\right\}
$$

Then the problem (ASP) satisfies the condition $\left\langle R_{3}\right\rangle$. Hence from Corollary 2.3, we get

$$
f_{i}=\underset{j \in D(i)}{\operatorname{Opt}}\left[\frac{t_{i j}+f_{j}}{1+t_{i j} f_{j}}\right] \quad i \notin S^{t}, \quad f_{i^{*}}=k\left(i^{*}\right) \in R \quad i^{*} \in S^{t} .
$$

For $0(=R(0)),(2)$ holds. So, in case $S^{t}=\{N\}$, from (8), we have

$$
f_{i}=\underset{j \in D(i)}{\mathrm{Opt}}\left[\frac{t_{i j}+f_{j}}{1+t_{i j} f_{j}}\right] \quad i \neq N, \quad f_{N}=0 .
$$


REMARK. One parameter associative operation which contains the operation: $a \circ$ $b=(a+b) /(1+a b)$ is as follows:

$$
a \circ b=g(s ; a, b)=\frac{a+b+(s-2) a b}{1+(s-1) a b} .
$$

Klir and Folger (1988) and Butnariu and Klement (1993) referred to this operation in their monographs. Under some condition on $t_{i j}$ we have recursive equations with one parameter $s$ which are similar to (14), (15).

In all examples stated above, $\left\{f_{i} \mid i \in V\right\}$ is a solution of the recursive equation (4). In what follows, we illustrate some examples in which (ASP) does not satisfy the condition $<R_{1}>$; hence it follows from Theorem 2.1 that $\left\{f_{i} \mid i \in V\right\}$ is not a solution of (4).

EXAMPLE 2.7. In this example we show that the problem (ASP) with the same network satisfies or does not satisfy the condition $\left\langle R_{1}\right\rangle$, depending on the choice of optimizer. Let us consider the fractional shortest path problem $(a \circ b=(a+b) /(1+a b)$, Opt $=$ min) on a network given in Fig.1.

We have

$$
\begin{aligned}
& f_{2}=\left(1 \circ \frac{1}{2} \circ 0\right) \wedge(1 \circ 2 \circ 0) \wedge(1 \circ 2 \circ 0)=1 \\
& f_{3}=(1 \circ 2 \circ 0) \wedge(1 \circ 2 \circ 0) \wedge\left(\frac{1}{2} \circ 3 \circ 0\right)=1
\end{aligned}
$$

Hence we get

$$
\min \left\{t_{13} \circ a \mid a \in A_{3}\right\}=(2 \circ 1) \wedge(2 \circ 1) \wedge\left(2 \circ \frac{7}{5}\right)=\frac{17}{19}<1=\min _{j \in D(1)}\left[t_{1 j} \circ f_{j}\right],
$$

thus, this problem does not satisfy the condition $\left\langle R_{1}\right\rangle$.

On the other hand, if we consider the fractional longest path problem $(a \circ b=$ $(a+b) /(1+a b), \mathrm{Opt}=\mathrm{Max})$ on the network given in Fig.1, then the problem satisfies the condition $\left\langle R_{1}\right\rangle$. In fact, we have

$$
f_{2}=1 \vee 1 \vee 1=1, \quad f_{3}=1 \vee 1 \vee \frac{7}{5}=\frac{7}{5}
$$

Hence we get

$$
\operatorname{Max}_{j \in D(1)}\left[t_{1 j} \circ f_{j}\right]=[2 \circ 1] \vee\left[2 \circ \frac{7}{5}\right]=1 \vee \frac{17}{19}=1 .
$$

Therefore,

$$
\operatorname{Max}_{j \in D(1)}\left[t_{1 j} \circ f_{j}\right]=1=\operatorname{Max}\left\{t_{12} \circ a \mid a \in A_{2}\right\}=\operatorname{Max}\left\{t_{13} \circ a \mid a \in A_{3}\right\} .
$$

Similarly, we can show that

$$
\operatorname{Max}_{j \in D(i)}\left[t_{i j} \circ f_{j}\right] \geq \operatorname{Max}\left\{t_{i j_{1}} \circ a \mid a \in A_{j_{1}}\right\} \text { for all } j_{1} \in D(i), i \neq 1,
$$

thus, this problem satisfies the condition $\left\langle R_{1}>\right.$. 


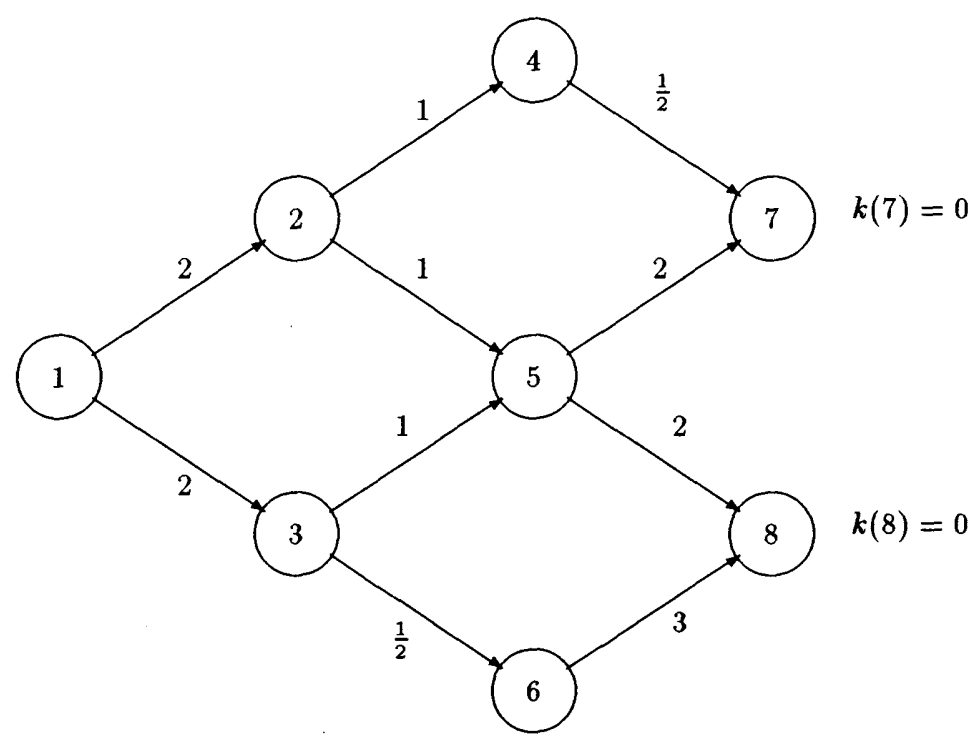

Figure 1: directed network

In view of Example 2.7, we introduce the following notation: $A_{s}(\min )$ and $A_{s}(\mathrm{Max})$ denote all problems satisfying the condition $\left\langle R_{1}\right\rangle$ in case Opt $=\min$ and Opt $=$ Max, respectively.

If the problem (ASP) satisfies the condition $\left\langle R_{3}\right\rangle$, then it belongs to both $A_{s}(\min )$ and $A_{s}(\operatorname{Max})\left((\operatorname{ASP}) \in A_{s}(\min ) \cap A_{s}(\operatorname{Max})\right)$. Therefore, all the problems which we considered in Examples $2.4 \sim 2.6$ belong to both. On the other hand, the problem in Example 2.7 belongs to $A_{s}(\mathrm{Max})$ but does not belong to $A_{s}(\mathrm{~min})$. Similarly, we can take an example of the problem satisfying that (ASP) $\in A_{s}(\mathrm{~min})$ and (ASP) $\notin A_{s}$ (Max). Further, in the following example, we illustrate a problem which belongs to neither $A_{s}(\mathrm{~min})$ nor $A_{s}(\mathrm{Max})$.

EXAMPLE 2.8. Let us replace only the arc length $t_{24}$ with $\frac{1}{2}$ in the network given in Fig.1. Then we consider the fractional problem: $a \circ b=(a+b) /(1+a b)$ on the changed network.

If Opt $=\min$, then we obtain

$$
\begin{aligned}
& f_{2}=\left(\frac{1}{2} \circ \frac{1}{2}\right) \wedge(1 \circ 2) \wedge(1 \circ 2)=\frac{4}{5} \wedge 1 \wedge 1=\frac{4}{5} \\
& f_{3}=(1 \circ 2) \wedge(1 \circ 2) \wedge\left(\frac{1}{2} \circ 3\right)=1 \wedge 1 \wedge \frac{7}{5}=1
\end{aligned}
$$

Hence we have

$$
\min \left\{t_{13} \circ a \mid a \in A_{3}\right\}=(2 \circ 1) \wedge\left(2 \circ \frac{7}{5}\right)=\frac{17}{19}<1=\min _{j \in D(1)}\left[t_{1 j} \circ f_{j}\right]
$$


thus, this problem does not satisfy the condition $\left\langle R_{1}\right\rangle$ in case Opt $=$ min. Consequently, it does not belong to $A_{s}$ (min).

Similarly we can show that this problem does not satisfy the condition $\left\langle R_{1}\right\rangle$ in case Opt $=$ Max, thus, it does not belong to $A_{s}(\operatorname{Max})$.

From now on, we consider only the problem which belongs to $A_{s}(\mathrm{Max})$ or $A_{s}(\mathrm{~min})$; hence we do not touch on such a problem as stated in Example 2.8. With this in mind, we will next discuss the existence and uniqueness of the solution of (4).

Lemma 2.9. Let $\left\{a_{j}\right\}_{j \in J},\left\{b_{j}\right\}_{j \in J},\left\{c_{j}\right\}_{j \in J} \subset R$ and put $d=\mathrm{Opt}_{j \in J}\left[a_{j} \circ b_{j}\right]$, $e=\mathrm{Opt}_{j \in J}\left[a_{j} \circ c_{j}\right]$, where $\circ$ is an associative operator and $J$ is a finite indexing set. Then there exists an index $j \in J$ such that

$$
|d-e| \leq\left|a_{j} \circ b_{j}-a_{j} \circ c_{j}\right| .
$$

Moreover, if $b_{j} \neq c_{j}$, then there exists a positive number $\alpha_{j}$ satisfying that

$$
|d-e| \leq \alpha_{j}\left|b_{j}-c_{j}\right|
$$

Proof. Take $k$ and $l$ satisfying

$$
d=a_{k} \circ b_{k}, \quad e=a_{l} \circ c_{l} .
$$

Then we have

$$
d \leq a_{l} \circ b_{l}, e \frac{\leq}{>} a_{k} \circ c_{k} .
$$

From (18) and (19), we obtain

$$
a_{k} \circ b_{k}-a_{k} \circ c_{k} \leq d-e \frac{\leq}{>} a_{l} \circ b_{l}-a_{l} \circ c_{l} .
$$

This leads us to

$$
|d-e| \leq\left|a_{l} \circ b_{l}-a_{l} \circ c_{l}\right| \vee\left|a_{k} \circ b_{k}-a_{k} \circ c_{k}\right| .
$$

Take $j$ satisfying that

$$
\left|a_{l} \circ b_{l}-a_{l} \circ c_{l}\right| \vee\left|a_{k} \circ b_{k}-a_{k} \circ c_{k}\right|=\left|a_{j} \circ b_{j}-a_{j} \circ c_{j}\right| .
$$

Then (16) follows.

Furthermore if $b_{j} \neq c_{j}$, then $\left|b_{j}-c_{j}\right|>0$. Hence there exists a sufficiently large, positive number $\alpha_{j}$ satisfying that

$$
\left|a_{j} \circ b_{j}-a_{j} \circ c_{j}\right|<\alpha_{j}\left|b_{j}-c_{j}\right| .
$$

Combining this with (16), we obtain the inequality (17).

THEOREM 2.10 (EXISTENCE AND UNIQUNESS). Let the problem (ASP) belong to $A_{s}(\min )$ or $A_{s}(\operatorname{Max})$. Then there exists a unique solution of $(4)$, where optimizer in (4) is $\min$ and $\mathrm{Max}$ in case $(\mathrm{ASP}) \in A_{s}(\min )$ and $(\mathrm{ASP}) \in A_{s}($ Max $)$, respectively. 
PRoof. First, from the assumption and Theorem 2.1, it follows that $\left\{f_{i} \mid i \in V\right\}$ defined by (3) is one solution of (4), where all optimizers are min and Max if (ASP) $\in A_{s}(\min )$ and $A_{s}(\operatorname{Max})$, respectively.

Second let us show that there can not be two different solutions of (4). We suppose that $\left\{f_{i} \mid i \in V\right\}$ and $\left\{g_{i} \mid i \in V\right\}$ are two solutions of (4). Let $i \notin S^{t}$ be an arbitrary but fixed node and put

$$
J=D(i), d=f_{i}, e=g_{i}, a_{j}=t_{i j}, b_{j}=f_{j}, c_{j}=g_{j}
$$

Then it follows from Lemma 2.14 that there exists $j \in D(i)$ such that $(i, j) \in A$ and

$$
\left|f_{i}-g_{i}\right| \leq\left|t_{i j} \circ f_{j}-t_{i j} \circ g_{j}\right|
$$

Suppose that $f_{j} \neq g_{j}$. Then $j \notin S^{t}$. From this and (16) in Lemma 2.14, we obtain

$$
\left|f_{j}-g_{j}\right| \leq\left|t_{j k} \circ f_{k}-t_{j k} \circ g_{k}\right|, k \in D(j)
$$

If $f_{k}=g_{k}$, then from this inequality, it follows that $f_{j}=g_{j}$, which contradicts the assumption. Hence $f_{k} \neq g_{k}$. So it follows from (17) that there exists $\alpha_{k}>0$ such that

$$
\left|f_{j}-g_{j}\right| \leq \alpha_{k}\left|f_{k}-g_{k}\right|
$$

By continuing in the same manner, we obtain

$$
\begin{aligned}
\left|f_{j}-g_{j}\right| & \leq \alpha_{k} \alpha_{l}\left|f_{l}-g_{l}\right| \\
& \vdots \\
& \leq \alpha_{k} \alpha_{l} \cdots \alpha_{n}\left|f_{n}-g_{n}\right|
\end{aligned}
$$

where $(j, k, l, \ldots, n)$ is a path, $f_{l} \neq g_{l}, \ldots, f_{n} \neq g_{n}$, and $\alpha_{l}, \ldots, \alpha_{n}>0$. Since $G(V, A)$ contains no cycles, $n \in S^{t}$ for a finite number of repetitions. Hence $f_{n}=g_{n}=k(n)$, which contradicts $(22)$. Therefore we conclude that $f_{j}=g_{j}$. From this and (21), it follows that $f_{i}=g_{i}$. Since $i$ is arbitrary node of $V$, the two solutions are in fact identical, which completes the proof.

Through the recursive equation (4), we can define the minimum (maximum) decision function $\pi$ as follows:

$$
\pi(i)=\text { the node } j \in V \text { which attains the minimum(maximum) of r.h.s. of (4). }
$$

Hence, optimal decision function $\pi(\cdot)$ generate a shortest(longest) path $(i, \hat{j}, \hat{k}, \ldots, \hat{m}, \hat{n}) ; \hat{n} \in$ $S^{t}$ as follows:

$$
\hat{j}=\pi(i), \hat{k}=\pi(\hat{j}), \ldots, \hat{n}=\pi(\hat{m})
$$




\section{Successive approximation method}

In this section we generate a sequence which converges to the solution of the recursive equation (4); the solution found by using the sequence gives us the desired minimal or maximal path lengths for the reason that the optimal path lengths comprise a solution of (4) and the solution of it is unique.

The sequence $\left\{f_{i}^{(k)}(\cdot)\right\}, k=0,1,2, \ldots$, which converges to the solution of (4) can be described as follows:

$$
\begin{aligned}
& k=0: f_{i^{*}}^{(0)}=k\left(i^{*}\right) \quad i^{*} \in S^{t}, \quad f_{i}^{(0)} \in R \quad i \notin S^{t} .
\end{aligned}
$$

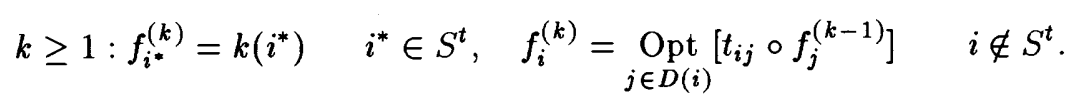

where $D(i)=\{j \in V \mid(i, j) \in A\}$.

Theorem 3.1 (Successive aPproximation). Suppose that the problem (ASP) belongs to $A_{s}(\min )$ or $A_{s}(\operatorname{Max})$. Let $\left\{f_{i}^{(k)}\right\}, k=0,1,2, \ldots$, be the sequence generated by $(23),(24)$. Then the sequence converges to the unique solution of (4), where optimizer in (4) and (24) is min and Max in case (ASP) $\in A_{s}(\min )$ and (ASP) $\in A_{s}($ Max), respectively.

Proof. Let $i \notin S^{t}$ be given but arbitrary. Let $k_{i}$ denote maximum of the number of arcs used when we move along paths from $i$ to the destination nodes and $k$ be fixed but arbitrary number such that $k_{i} \leq k$.

Then in the same way as in the proof of Theorem 2.15 , we can show that for each $i \notin S^{t}$ there exists a path

$$
p=(i, j(1), j(2), \ldots, j(l))
$$

such that

$$
\begin{aligned}
\left|f_{i}^{(k)}-f_{i}^{(k-1)}\right| & \leq\left|t_{i j(1)} \circ f_{j(1)}^{(k-1)}-t_{i j(1)} \circ f_{j(1)}^{(k-2)}\right| \\
& \leq \alpha_{j(2)} \alpha_{j(3)} \cdots \alpha_{j(l)}\left|f_{j(l)}^{(k-l)}-f_{j(l)}^{(k-l-1)}\right|
\end{aligned}
$$

where $\alpha_{j(\cdot)}>0$.

If $j(l) \in S^{t}$, then from (25), we obtain

$$
f_{i}^{(k)}=f_{i}^{(k-1)}
$$

In case $j(l) \notin S^{t}$, take $l=k_{i}-1$. Then since $D(j(l)) \subset S^{t}$, we have

$$
f_{j(l)}^{(k-l)}=f_{j(l)}^{(k-l-1)}=\operatorname{Opt}_{j \in D(j(l))}\left[t_{i j} \circ k(j)\right]=t_{i j^{*}} \circ k_{\left(j^{*}\right)}
$$

Hence, from (25) we see that (26) holds even if $j(l) \notin S^{t}$. Consequently, for each $i \notin S^{t}$

$$
f_{i}^{(k)}=f_{i}^{(k-1)}, \quad k=k_{i}, k_{i}+1, \ldots
$$


Hence, for each $i \notin S^{t}$ the sequence $\left\{f_{i}^{(k)}\right\}$ converges to the real number $f_{i}^{\left(k_{i}-1\right)}$. Put $\operatorname{Max}_{i \notin S^{t}} k_{i}=\bar{k}$. Then from (27) we obtain

$$
\begin{aligned}
& f_{i}^{(\bar{k})}=\underset{j \in D(i)}{\operatorname{Opt}}\left[t_{i j} \circ f_{j}^{(\bar{k}-1)}\right]=\underset{j \in D(i)}{\operatorname{Opt}}\left[t_{i j} \circ f_{j}^{(\bar{k})}\right] \quad i \notin S^{t}, \\
& f_{i^{*}}^{(\bar{k})}=k\left(i^{*}\right) \quad i^{*} \in S^{t} .
\end{aligned}
$$

Therefore, $\left\{f_{i}^{(\bar{k})} \mid i \in V\right\}$ is the solution of (4).

REMARK. Let $\bar{k}$ be the same number as in the proof in Theorem 3.1 and $N$ be potency of the set $V$. Then since $\bar{k} \leq N-1$, the sequence generated by (23), (24) converges in at most $N-1$ steps.

CoRollary 3.2. Let the problem (ASP) satisfy the assumption of Theorem 3.1. Let $\left\{f_{i}^{(k)}\right\}$ be the sequence generated by (24) and the following:

$$
k=0: f_{i^{*}}^{(0)}=k\left(i^{*}\right) \quad i^{*} \in S^{t}, \quad f_{i}^{(0)}=\underset{j \in D(i)}{\operatorname{Opt}} t_{i j} \quad i \notin S^{t}
$$

Then the sequence converges to the unique solution of (4), where optimizer in (4), (24) and (28) is $\min$ and $\mathrm{Max}$ in case (ASP) $\in A_{s}(\min )$ and (ASP) $\in A_{s}($ Max $)$, respectively.

Proof. Since for $i \notin S^{t}, f_{i}^{(0)}=\mathrm{Opt}_{j \in D(i)} t_{i j} \in R$, it follows from Theorem 3.1 that the sequence generated by (24) and (28) converges to the solution of (4).

CoRollary 3.3. Let the problem (ASP) satisfy the following condition:

(a) $k\left(i^{*}\right)=R(0) \quad i^{*} \in S^{t}$,

(b) each $t_{i j}$ and $R(\circ)$ belong to a set $A^{\prime}$ such that $\left(A^{\prime}, \circ\right)$ is a semigroup(, that is, $\circ: A^{\prime} \times A^{\prime} \rightarrow A^{\prime}$, associative operation),

(c) the associative operation is monotone on $A^{\prime} \subset R$ at each $t_{i j}, i, j \notin S^{t}$ :

$$
a, b \in A^{\prime}, a \leq b \Longrightarrow t_{i j} \circ a \leq t_{i j} \circ b
$$

Then the meaning of $f_{i}^{(n)}$ generated by (28) and (24) is as follows:

$$
\begin{aligned}
f_{i}^{(n)}= & \text { the length of the shortest(longest) path from node } i \text { to reachable node } \\
& \text { or to } i^{*} \in S^{t} \text { when } n+1 \text { or fewer arcs are used, respectively, }
\end{aligned}
$$

where the length of a path $\left(i, j_{1}, j_{2}, \ldots, j_{k}, j\right)$ from $i$ to $j \notin S^{t}$ is defined by $t_{i j_{1}} \circ t_{j_{1} j_{2}} \circ$ $\cdots \circ t_{j_{k} j}$.

Proof. We will show only the case Opt $=\min$. In the same manner, the case Opt $=$ Max is proved. From (28) and (a), (29) holds for $n=0$. Assume (29) is true for $n=k-1$. Then We will show the same for $n=k$. 
Suppose that there exists a path $\left(i, j_{1}, j_{2}, \ldots, j_{l}, j_{l+1}\right), l \leq k$ such that

$$
f_{i}^{(k)}>t_{i j_{1}} \circ t_{j_{1} j_{2}} \circ \cdots \circ t_{j_{i} j_{1+1}} \text {. }
$$

where in case $l<k$, then $j_{l+1} \in S^{t}$. It follows from (24) that

$$
t_{i j_{1}} \circ f_{j_{1}}^{(k-1)} \geq f_{i}^{(k)} \text {. }
$$

Hence from (30), we have

$$
t_{i j_{1}} \circ f_{j_{1}}^{(k-1)}>t_{i j_{1}} \circ t_{j_{1} j_{2}} \circ \cdots \circ t_{j_{l} j_{l+1}} .
$$

Therefore it follows from (b), (c) and the inductive assumption that

$$
f_{j_{1}}^{(k-1)}>t_{j_{1} j_{2}} \circ \cdots \circ t_{j_{1} j_{l+1}}
$$

where in case $l<k$, then $j_{l+1} \in S^{t}$. This contradicts the assumption of $f_{j_{1}}^{(k-1)}$. Consequently, we get

$$
f_{i}^{(k)} \leq \min _{p, l \leq k}\left[t_{i j_{1}} \circ t_{j_{1} j_{2}} \circ \cdots \circ t_{j_{l} j_{l+1}}\right]
$$

where $p=\left(i, j_{1}, j_{2}, \ldots, j_{l}, j_{l+1}\right)$.

On the other hand, the reverse inequality to (32) follows from the definition of $f_{i}^{(k)}$ and the inductive assumption. Consequently, (29) also holds for $n=k$, which completes the proof.

Let

$$
\begin{aligned}
\pi^{(k)}(i)= & \text { the node } j \in V \text { which attains the minimum(maximum) of r.h.s. } \\
& \text { of the second equation in (24). }
\end{aligned}
$$

Then in the same way as in Section 2, optimal decision function $\pi^{(k)}(\cdot)$ generates the shortest(longest) path from node $i$ to reachable node or to destination node when $k+1$ or fewer arcs are used, respectively.

EXAMPLE $3.4(a \circ b=(a+b) /(1+a b)$, Opt $=\min )$. We consider an example of a network given in Fig.2. The sequence $\left\{f_{i}^{(k)}\right\}, k=0,1,2, \ldots$, can be computed successively as shown in Table 1 . Since $f_{i}^{(2)}=f_{i}^{(k)}, k \geq 3, i=1 \sim 8$, we obtain $f_{i}=f_{i}^{(2)}, i=1 \sim 8$. We remark that since this problem satisfies the assumptions of Corollary 3.3, each $f_{i}^{(k)}$ has such meaning as in (29). For each $i$ the node $\pi^{(k)}(i)$ is presented in Table 2. Using the optimal decision function $\pi^{(k)}(\cdot)$, we can determine the shortest path $(1,2,5,7)$. 
Table 1:

\begin{tabular}{c|c|c|c|c}
\hline Node & $f_{i}^{(0)}$ & $f_{i}^{(1)}$ & $f_{i}^{(2)}=f_{i}$ & $f_{i}^{(3)}$ \\
\hline 1 & $\frac{1}{3}$ & $\frac{7}{13}$ & $\frac{11}{13}$ & $\frac{11}{13}$ \\
2 & $\frac{1}{4}$ & $\frac{5}{7}$ & $\frac{5}{7}$ & $\frac{5}{7}$ \\
3 & $\frac{1}{4}$ & $\frac{2}{3}$ & $\frac{2}{3}$ & $\frac{2}{3}$ \\
4 & 2 & 2 & 2 & 2 \\
5 & $\frac{1}{2}$ & $\frac{1}{2}$ & $\frac{1}{2}$ & $\frac{1}{2}$ \\
6 & 3 & 3 & 3 & 3 \\
7 & 0 & 0 & 0 & 0 \\
8 & 0 & 0 & 0 & 0 \\
\hline
\end{tabular}

Table 2:

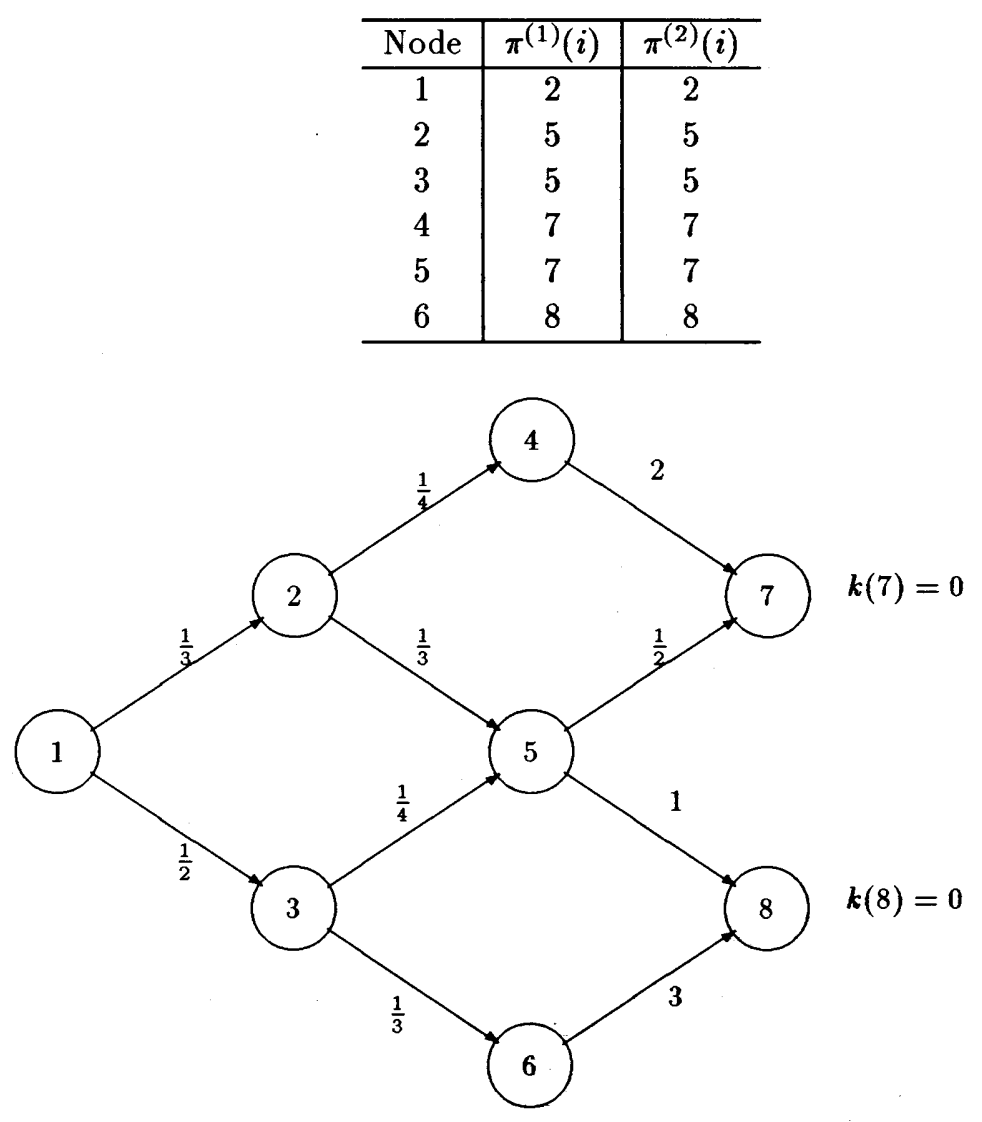

Figure 2: directed network 


\section{Acknowledgement}

The author would like to thank the referee for his several suggestions, which improve the first manuscript.

\section{References}

Bellman, R., Esogbue, A.O. and Nabeshima, I. (1982) Mathematical Aspects of Scheduling and Applications, Pergamon Press, New York.

Butnariu, D. and Klement, E.P. (1993) Triangular norm-based measures and games with fuzzy coalitions, Kluwer Academic Publishers.

Dreyfus, S.E. (1969) An appraisal of some shortest-path algorithms, Oper. Res.,17, 395-412.

Iwamoto, S. (1987) Theory of Dynamic Programs (in Japanese), Kyushu University Press.

Iwamoto, S. (1996) Associative dynamic programs, J. Math. Anal. Appl., 201, 195-211.

Klir, G.J. and Folger, T.A. (1988) Fuzzy sets, uncertainty and information, Prentice Hall.

Maruyama, Y. (1996) Shortest and longest path problems, Optimization, 38, 287-299.

Smith, D.K. (1991) Dynamic Programming, Ellis Horwood Limited.

Sniedovich, M. (1992) Dynamic Programming, Marcel Dekker.

Received June 12, 1996

Revised October 7, 1996 